\title{
Extreme statistics of intensity fluctuations in nonequilibrium steady states
}

\author{
G. Györgyi ${ }^{\dagger}$, P. C. W. Holdsworth*, Z. Rácz ${ }^{\dagger}$, and B. Portelli* \\ †Institute for Theoretical Physics, HAS Research Group, Eötvös University, Hungary \\ gyorgyi@glu.elte.hu, racz@general.elte.hu \\ *Laboratoire de Physique, Ecole Normale Supérieure, Lyon, France \\ pcwh@ens-lyon.fr, Baptiste.Portelli@ens-lyon.fr
}

\begin{abstract}
Stochastic surface growth driven by surface tension (Edwards-Wilkinson model) is investigated. The much studied stationary state, characterized by Gaussian distributed Fourier modes with power-law dispersion, is reexamined here to include extremal value statistics. We calculate the probability distribution of the largest Fourier intensity and find that, generically, it does not obey any of the known extreme statistics limit distributions, apart from special border cases where the Fisher-Tippett-Gumbel (FTG) distribution emerges. If a gap is, however, introduced in the dispersion then necessarily the FTG distribution is recovered.
\end{abstract}

Keywords: Nonequilibrium stationary distributions, extreme statistics, scaling functions

\section{MOTIVATION AND OUTLINE}

Extreme value statistics ${ }^{1}$ (EVS) has recently been used as a tool in the analysis of a number of physical systems such as spin glasses, ${ }^{2}$ travelling fronts, ${ }^{3}$ granular materials, ${ }^{4}$ etc. In most of the examples studied, EV $\$$ emerges in response to "which-is-the-largest" type questions. There are, however, cases where the connection to EVS is much less understandable. For example, non-Gaussian fluctuations of macroscopic quantities in strongly fluctuating systems (both experimental ${ }^{5}$ and model systems ${ }^{6}$ ) have been observed to obey distributions which bare strong resemblance to the Fisher-Tippett-Gumbel (FTG) distribution, one of the limiting distributions of extremal statistics. ${ }^{7}$ In particular, interface fluctuations ${ }^{8-11}$ produce roughness distributions which are exactly the FTG distribution for $d=1$ dimensional periodic interfaces equivalent to Gaussian $1 / f$ noise. Furthermore, the fluctuations of the Edwards-Wilkinson interface ${ }^{12}$ in $d=2$ can be fit to a generalized FTG distribution. ${ }^{6}$

In order to clarify the possible connection between the fluctuations of macroscopic quantities in strongly fluctuating (quasi-critical) systems and EVS, we undertook a detailed study ${ }^{13}$ of the Edwards-Wilkinson typ interface growth. $^{12}$ In this process, the probability of local growth is governed by surface tension and the stationary probability distribution $\mathcal{P}[h(\boldsymbol{x})]$ of interface configurations $h(\boldsymbol{x})$ (height of the interface above the substrate at coordinate $\boldsymbol{x})$ is a simple quadratic functional $\mathcal{P}[h(\boldsymbol{x})] \propto \exp (-S[h(\boldsymbol{x})])$ with the action given by $S=\sigma \int \mathrm{d}^{d} x|\nabla h(\boldsymbol{x})|^{2}$ (here $\sigma$ is the surface tension coefficient and $d$ is the dimensionality of the substrate). The surfaces described by $\mathcal{P}[h(\boldsymbol{x})]$ are rough for $d \leq 2$, meaning that the mean-square height fluctuations diverge in the infinite-system limit. This "criticality" can be understood in terms of the divergence of long-wavelength fluctuations which cannot be constrained by surface tension. The divergence of the amplitude of the longwavelength modes suggests the idea that the largest amplitude Fourier modes may dominate the roughness distribution and this may open up a way to connect EVS and the distribution of macroscopic quantities such as roughness. The above idea led us to study the extreme statistics of the intensities (square modulus of Fourier coefficients of the height function) in the Edwards-Wilkinson model.

Since $S[h(\boldsymbol{x})]$ is a sum of contributions from the independent Fourier modes, we can actually carry out the calculations for a generalized Edwards-Wilkinson model where an arbitrary power-law dispersion is used, i.e. $S=\sigma \sum_{\boldsymbol{k}}|\boldsymbol{k}|^{\alpha}\left|c_{\boldsymbol{k}}\right|^{2}$, where $c_{\boldsymbol{k}}$ is the amplitude of the Fourier mode of wavenumber $\boldsymbol{k}$ and $\left|c_{\boldsymbol{k}}\right|^{2}$ is the corresponding intensity. The significance of this generalization goes beyond the original motivation of real $2 \mathrm{~d}$

Mailing address: Institute for Theoretical Physics, Eötvös University, Pázmány sétány 1/a, 1117 Budapest, Hungar. 
surfaces. For $d=1$ it gives the path distribution for $1 / f^{\alpha}$ Gaussian processes, in particular it represent Brownian motion for $\alpha=2$, and $1 / f$ noise for $\alpha=1$. We also consider another generalization which leads to $a$ remarkably simple and general result. It is the introduction of a gap in the dispersion $S_{r}=\sigma \sum_{\boldsymbol{k}}\left[r+|\boldsymbol{k}|^{\alpha}\right]\left|c_{\boldsymbol{k}}\right|^{2}$ which corresponds to surface fluctuations in an external field (and also well known in the field theory of critical phenomena as representing the high-temperature phase).

\section{RESULTS}

It follows from the action, $S$, that the intensities $\left|c_{\boldsymbol{k}}\right|^{2}$ are exponentially distributed and are independent of each other. Thus a relatively simple calculation provides the probability $P(z) d z$ that the extremal intensity is in the interval $z \leq\left|c_{\boldsymbol{k}}\right|_{\text {max }}^{2} \leq z+d z$. Apart from scale factors (for details see our paper ${ }^{13}$ ), $P(z)$ is given by

$$
P_{\alpha}(z)=\prod_{n=1}^{\infty}\left(1-e^{-n^{\alpha} z}\right) \sum_{n=1}^{\infty} \frac{n^{\alpha}}{e^{n^{\alpha} z}-1} .
$$

The actual form of the functions for various $\alpha$ is displayed on Fig.1 where the scale factors were taken care by scaling the maximal intensity by its average value $\langle z\rangle$.

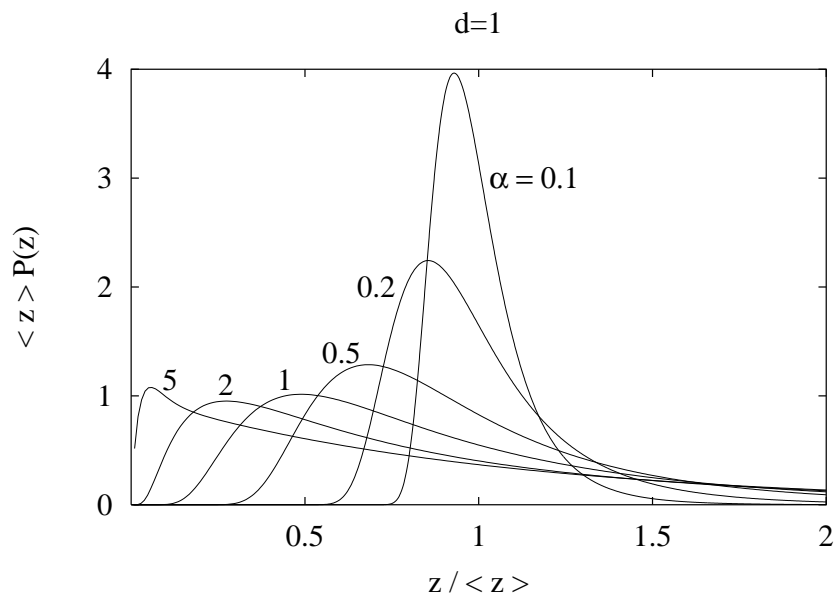

Figure 1. Maximal intensity $\left(z=\left\langle\left|c_{\boldsymbol{k}}\right|_{\text {max }}^{2}\right\rangle\right)$ distributions for generalized one-dimensional Edwards-Wilkinson interfaces The scaled probability of the maximal intensity is plotted against the scaled intensity. Gapless $(r=0)$ dispersion of index $\alpha$ is considered.

An inspection of the expression for $P(z)$ reveals that it is none of the classic known ${ }^{7}$ limit distribution $\$$ of EVS. Thus the idea of largest amplitude modes dominating the roughness distribution is not valid for the Edwards-Wilkinson model with gapless power-law dispersion. There are, however, border cases where the FTC distribution does emerge. In the limit $\alpha \rightarrow 0$, where the intensities become identically distributed, this result is obvious. Less obvious is that FTG also emerges in the limits where the dimensionality of the system goes to infinity or only the short-wavelength modes are kept in the system. Finally, in the presence of a gap in the dispersion $(r \neq 0)$, we find ${ }^{14}$ that the maximal intensity distribution is the FTG distribution independently of $o$ and $d$.

In conclusion we would like to emphasize a few propositions. First, in most experimental setups the Fourie: intensity statistics is usually straightforward to measure, not only on surfaces but for any data stream. Thus the distribution functions displayed above should be experimentally accessible. Secondly, while our model had independent Fourier modes, some qualitative results may be more generally valid. Such features could be the single-hump shape of the PDFs of extremal modes, the absence of the FTG distribution for generic gaples\$ dispersions, as well as the appearance of FTG, if there is a gap, and in some limiting cases without a gap, like high dimensions or weak dispersion. 


\section{ACKNOWLEDGMENTS}

This work has been partially supported by the Hungarian Academy of Sciences (Grant T043734) and by the CNRS ACI (Grant No.2226).

\section{REFERENCES}

1. E. J. Gumbel, Statistics of Extremes, Columbia University Press, 1958.

2. J.-P. Bouchaud and M. Mézard, "Universality classes for extreme-value statistics," J. Phys. A: Math. Gen. 30, pp. 7997-8015, 1997.

3. D. S. Dean and S. N. Majumdar, "Extreme-value statistics of hierarchically correlated variables deviation from Gumbel statistics and anomalous persistence," Phys. Rev. E 64, pp. 046121 1-5, 2001.

4. G. D'Anna, P. Mayor, G. Gremaud, A. Barrat, and V. Loreto, "Extreme events-driven glassy behaviour in granular media," Europhys. Lett. 61, pp. 60-66, 2003.

5. S. T. Bramwell, P. C. W. Holdsworth, and J.-F. Pinton, "Universality of rare fluctuations in turbulence and critical phenomena," Nature 396, pp. 552-554, 1998.

6. S. T. Bramwell, K. Christensen, J.-Y. Fortin, P. C. W. Holdsworth, H. J. Jensen, S. Lise, J. M. López, M. Nicodemi, J.-F. Pinton, and M. Sellitto, "Universal fluctuations in correlated systems," Phys. Rev. Lett 84, pp. 3744-3747, 2000.

7. S. Coles, An Introduction to Statistical Modeling of Extreme Values, Springer Verlag, London, 2001.

8. G. Foltin, K. Oerding, Z. Rácz, R. L. Workman, and R. K. P. Zia, "Width distribution for random-walk interfaces," Phys. Rev. E 50, pp. 1087-1091, 1994.

9. Z. Rácz and M. Plischke, "Width distribution for (2+1)-dimensional growth and deposition processes," Phys. Rev. E 50, pp. 3530-3537, 1994.

10. T. Antal and M. Droz and G. Györgyi and Z. Rácz, "1/f noise and extreme value statistics," Phys. Rev. Lett. 87, pp. 240601 1-4, 2001.

11. T. Antal, M. Droz, G. Györgyi, and Z. Rácz, "Roughness distributions for $1 / f^{\alpha}$ signals," Phys. Rev. E 65 , pp. 046140 1-12, 2002.

12. A.-L. Barabási and H. E. Stanley, Fractal Concepts in Surface Growth, Cambridge University Press, Cambridge, 1995.

13. G. Györgyi, P. Holdsworth, Z. Rácz, and B. Portelli, "Statistics of extremal intensities for Gaussian interfaces," Phys. Rev. E 68, pp. 056116 1-14, 2003.

14. G. Györgyi, P. Holdsworth, and Z. Rácz unpublished. 